\section{Resultados en el tratamiento de pacientes con leucemia mieloide aguda no promielocítica en el Hospital Clínico de la Pontificia Universidad Católica entre los años 2010-2014}

\author{
MÓNICA FUENTES ${ }^{a}$, PATRICIO ROJAS ${ }^{\text {a }}$, \\ DANIEL ERNST, MAURICIO OCQUETEAU, PABLO BERTIN, \\ MAURICIO SARMIENTO, PABLO RAMÍREZ ${ }^{1}$
}

\section{Results of acute myeloid leukemia treatment. Analysis of 63 patients between 2010-2014}

Background: Acute myeloid leukemia (AML) is the most common acute leukemia in adults, emphasizing its high recurrence rate despite hematopoietic cell transplantation (HCT). Aim: To report the results of AML treatment at the Catholic University of Chile Clinical Hospital. Patients and Methods: Review of medical records of patients with AML. Results: 63 patients, median age 55.4 years (range:16-89), treated between 2010 and 2014. Admission laboratory values showed (median values): leukocytes $45.989 / \mathrm{mm}^{3}$, hemoglobin $9.1 \mathrm{~g} / \mathrm{dl}$, platelets $75.548 / \mathrm{mm}^{3}$, peripheral blood blasts $38 \%$ and bone marrow blasts $74 \%$. According to cytogenetic risk classification we observed the following groups: favorable $8 \%(n=5)$, intermediate $51 \%(n=32)$, unfavorable $13 \%(n=8)$ and unknown $28 \%(n=17)$. Seventy five percent of patients received induction chemotherapy and $25 \%$ palliative care. Median survival of treated and palliative care patients was 27.3 and 1 month respectively. Induction chemotherapy (IC) mortality (ICM) was $4.2 \%$. Seventy percent $(n=33)$ of patients who received IC had complete response (CR) with a 3-year relapse free survival (RFS) of 25\% and overall survival (OS) of $31 \%$. Multivariate analysis demonstrated that achievement of $C R$, cytogenetic risk group and receiving consolidation chemotherapy were significantly associated with better RFS and OS. Conclusions: AML treatment with standard chemotherapy in our center achieves similar results to what has been described in international series regarding induction rates and ICM, however RFS and OS are still very low, especially in intermediate and high cytogenetic risk groups.

(Rev Med Chile 2015; 143: 1269-1276)

Key words: Antineoplastic protocols; Drug therapy; Leukemia, myeloid, acute.

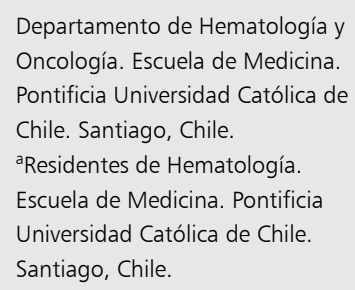

Recibido el 26 de marzo de 2015, aceptado el 23 de julio de 2015.

\section{Correspondencia a:}

Dr Pablo Ramírez Villanueva

Dirección: Lira \# 85, $4^{\circ}$ piso.

Santiago.

Teléfono: 223543092

pramirez@med.puc.cl

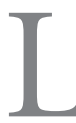

a leucemia mieloide aguda no promielocítica (LMA) es la leucemia más frecuente del adulto correspondiendo aproximadamente a $80 \%$ del total de las leucemias agudas de los adultos. En Estados Unidos de Norteamérica y Europa la incidencia aproximada es de 3-5 casos por 100.000 habitantes ${ }^{1,2}$ y la edad de presentación promedio es de 65 años ${ }^{1}$. En nuestro país no hay datos claros al respecto.

Según marcadores clínicos, moleculares y cariotipo se establecen diferentes grupos de riesgo que difieren en sobrevida global (SG) lo que ha 
permitido un mejor enfrentamiento terapéutico de los pacientes ${ }^{3}$.

El tratamiento de la LMA se divide en quimioterapia (QT) de inducción de la remisión y terapia de postremisión. La remisión completa (RC) citológica se alcanza en aproximadamente $70-80 \%$ de los menores de 60 años y 30-50\% en aquellos mayores de 60 años con buen estado funcional. Una vez lograda la remisión, es necesario iniciar el tratamiento de postremisión o "consolidación" para prevenir recaídas. No obstante los avances en esquemas QT de inducción altamente efectivos en conseguir RC, la SG de LMA en adultos es baja: menos de $50 \%$ a 5 años en pacientes menores de 45 años y menos de $5 \%$ en el grupo mayor de 65 años. En niños, la SG ha aumentado a $60 \%$ a 5 años 2 .

En Chile hay escasa información publicada sobre de los resultados del tratamiento de LMA en población adulta, siendo la única serie proveniente la del Hospital del Salvador de el año $2000^{4}$ en la que se describen 117 enfermos con respuesta completa a la inducción en $65 \%$ de éstos ${ }^{3}$. En el presente trabajo se describen los resultados del tratamiento de pacientes con LMA diagnosticados y tratados en la Pontificia Universidad Católica de Chile y su comparación con series internacionales y local.

\section{Pacientes y Métodos}

\section{Población en estudio}

Se realizó un análisis retrospectivo de la base de datos de LMA del Departamento de Hematología Oncología y los registros electrónicos e historias clínicas y se obtuvieron datos sobre características epidemiológicas, clínicas y de laboratorio de los pacientes con LMA mayores de 16 años que fueron diagnosticados y tratados en el Hospital Clínico de la Pontificia Universidad Católica de Chile entre los años 2010 y 2014.

\section{Diseño de estudio y colección de datos}

Se revisaron variables clínicas y de laboratorio incluyendo entre otras edad, hemograma al ingreso, porcentaje de blastos en sangre periférica, porcentaje de blastos en mielograma. Según la citogenética de los pacientes se los clasificó en 3 grupos de riesgo de acuerdo a la clasificación MRC revisada ${ }^{4,5}$ : favorable, intermedio y desfavorable. Dado que no hubo un estudio molecular consistente a todos los pacientes al momento del diagnóstico, no se incluyó el análisis en el presente estudio.

Se consignó la quimioterapia de inducción (QT) recibida en 3 subtipos: “7+3" estándar (daunorrubicina $60-90 \mathrm{mg} / \mathrm{m}^{2} /$ día iv por 3 días y citarabina $100-200 \mathrm{mg} / \mathrm{m}^{2} / \mathrm{d}$ iv por 7 días); " $7+3$ " idarrubicina (idarrubicina $12 \mathrm{mg} / \mathrm{m}^{2} /$ día iv por 3 días y citarabina $100-200 \mathrm{mg} / \mathrm{m}^{2} /$ día iv por 7 días ); azaciticina en dosis de $75 \mathrm{mg} / \mathrm{m}^{2} /$ día sc por 7 días. La respuesta citológica a la inducción se definió según criterios de la International Working Group ${ }^{5,6}$ que, brevemente, clasifica la respuesta en remisión completa $(\mathrm{RC}):<5 \%$ de blastos en médula ósea, ausencia de bastones de Auer, ausencia de compromiso extra medular, RAN $>1.000$, recuento plaquetario $>100.000 \mathrm{x}$ $\mathrm{mm}^{3}$, independencia de transfusión de glóbulos rojos; remisión completa con recuperación incompleta (RCI): cumple con criterios de RC, excepto RAN $<1.000$, recuento plaquetario $<100.000 \mathrm{x}$ $\mathrm{mm}^{3}$; estado morfológico libre de leucemia: $<5 \%$ blastos en médula ósea, ausencia de bastones de Auer, ausencia de compromiso extra medular, sin recuperación hematológica; remisión parcial (RP): relevante en ensayos clínicos fase 1 o 2; recaída: blastos en médula $>5 \%$ o reaparición de blastos en sangre periférica o desarrollo de compromiso extra medular; refractariedad (resistencia): falla en lograr RC en la inducción. Esto se conoce también como falla de inducción. Los pacientes que requieren dos ciclos de quimioterapia para lograr RC no se consideran refractarios.

La quimioterapia de consolidación se indicó en ciclos de cada 28 días, a los pacientes que lograron la RC y los tipos usados fueron: citarabina en altas dosis (HiDAC) (1-3 g/cada $12 \mathrm{~h}$ iv por 6 dosis, en días alternos o consecutivos, 2 a 4 ciclos totales), azacitidina $75 \mathrm{mg} / \mathrm{m}^{2} /$ día sc por 7 días (cada 21-28 días por hasta 6 ciclos según la respuesta); esquema "HAM" (mitoxantrona $5 \mathrm{mg} / \mathrm{m}^{2} / \mathrm{d}$ iv por 5 días y citarabina $500 \mathrm{mg} / \mathrm{m}^{2} / \mathrm{d}$ iv cada $12 \mathrm{~h}$ por 6 días, entre 1 y 3 ciclos de consolidación por paciente). Previo al inicio de éstos, se realizó mielograma y medición de EMR para objetivar que el paciente siguiera en RC.

En la evaluación de las complicaciones de los pacientes, se consignó la mortalidad en la QT de inducción (mortalidad durante la inducción) y la mortalidad dentro del seguimiento total de la población en estudio que fue de una total de 3 años. Para el estudio de neutropenia febril, nos basamos en las definiciones de las guías IDSA $2010^{6}$. 
Dentro del seguimiento del grupo en estudio, se realizó análisis de la sobrevida libre de recaída (SLR) y SG según definiciones epidemiológicas internacionales ${ }^{7}$.

\section{Análisis estadístico}

Para el análisis de los datos se usó el programa informático estadístico IBM SPSS Statistics $22.0 \mathrm{v}$ 2.1 (New York, NY. USA) El nivel de significancia para determinar establecer diferencias significativas fue basado en un error alpha $<0,05(\mathrm{p}<0,005)$. Las curvas de sobrevida representadas por Kaplan Meier y comparadas mediante Log Rank Test.

\section{Resultados}

\section{Características clínicas y demográficas}

Entre los años 2010 y 2014 se diagnosticaron 63 pacientes con LMA. De éstos, 52 fueron hombres
(66\%) con una edad media de 55,4 años (rango, 16-89). Destaca la leucocitosis, anemia y trombocitopenia moderada. El 9,5\% de los pacientes no presentaron blastos en sangre periférica al diagnóstico. El detalle de las características demográficas y de laboratorio se resumen en la Tabla 1.

La distribución de los pacientes según riesgo citogenético al diagnóstico estratificó a los pacientes en grupo favorable $8 \%(n=5$, edad promedio 41 años); intermedio $51 \%(n=32$, edad promedio 58 años) y desfavorable $13 \%(\mathrm{n}=8$, edad promedio 41,5 años). El $28 \%$ de los pacientes no tuvo estudio citogenético $(n=16$, edad promedio 73,5 años) (Tabla 2).

\section{Quimioterapia de inducción}

En total, $75 \%(n=47)$ recibió QT de inducción que se dividió en 3 grupos: $83 \%(n=39)$ con esquema " $7+3$ " estándar, $11 \%(\mathrm{n}=5)$ con

Tabla 1. Características clínicas y de laboratorio de todos los pacientes con LMA y según si recibieron o no quimioterapia (QT) (test-t para muestras independientes, test exacto de Fisher)

\begin{tabular}{|c|c|c|c|c|}
\hline & LMA todos (n: 63) & LMA QT (n: 47) & LMA $\sin Q T(n: 16)$ & QT vs sin QT Valor $p$ \\
\hline Edad (años y rango) & $59,3(17-89)$ & $53,6(17-81)$ & $75,7(53-89)$ & $<0,001$ \\
\hline Género masculino & $67 \%(n=42)$ & $70 \%(n=33)$ & $56 \%(n=9)$ & 0,364 \\
\hline Leucocitos $\left(/ \mathrm{mm}^{3}\right)$ & $\begin{array}{c}46.000 \\
(700-405-000)\end{array}$ & $\begin{array}{c}41.917 \\
(700-405.500)\end{array}$ & $\begin{array}{c}57.694 \\
(800-212.900)\end{array}$ & 0,451 \\
\hline Blastos periferia & $38 \%(0-100)$ & $39 \%(0-100)$ & $36 \%(0-48)$ & 0,778 \\
\hline $\mathrm{Hb}(\mathrm{g} / \mathrm{dl})$ & $9,1(5,2-14,1)$ & $9,3(5,2-14,1)$ & $8,7(6,5-11,4)$ & 0,281 \\
\hline Plaquetas $\left(/ \mathrm{mm}^{3}\right)$ & $\begin{array}{c}76.000 \\
(10.000-454.000)\end{array}$ & $\begin{array}{c}82.152 \\
(10.000-454.000)\end{array}$ & $\begin{array}{c}48.560 \\
(11.000-112.000)\end{array}$ & 0,162 \\
\hline Fibrinógeno (mg/dl) & $391(160-606)$ & $392(219-606)$ & $387(160-580)$ & 0,897 \\
\hline Blastos médula ósea & $74 \%(5-100)$ & $76 \%(7-100)$ & $65 \%(20-100)$ & 0,342 \\
\hline \multicolumn{5}{|l|}{ Riesgo citogenético } \\
\hline Favorable & $(n=5)$ & $10,6 \%(n=5)$ & $0 \%$ & 0,317 \\
\hline Intermedio & $51 \% \quad(n=32)$ & $61,7 \% \quad(n=29)$ & $19 \% \quad(n=3)$ & 0,004 \\
\hline Desfavorable & $13 \% \quad(n=8)$ & $12,7 \% \quad(n=6)$ & $12,5 \%(n=2)$ & 1,000 \\
\hline Desconocido & $28 \% \quad(n=16)$ & $15 \% \quad(n=7)$ & $68,5 \%(n=11)$ & $<0,001$ \\
\hline
\end{tabular}

Tabla 2. Quimioterapia (QT) de inducción y consolidación administrada a los pacientes según riesgo citogenético

\begin{tabular}{|c|c|c|c|c|c|c|}
\hline \multirow{3}{*}{$\begin{array}{l}\text { Grupos citogenéticos } \\
\text { Favorable }\end{array}$} & \multicolumn{3}{|c|}{ QT inducción } & \multicolumn{3}{|c|}{ QT consolidación } \\
\hline & $7+3$ (n: 39) & otro (n: 8) & Valor-p & Sí (n: 36) & No (n: 11) & Valor-p \\
\hline & $5(13 \%)$ & $0 \quad(0 \%)$ & 0,571 & $5(14 \%)$ & $0 \quad(0 \%)$ & 0,322 \\
\hline Intermedio & $25(64 \%)$ & $4(50 \%)$ & 0,692 & $24(67 \%)$ & $5(45 \%)$ & 0,291 \\
\hline Desfavorable & $5(13 \%)$ & $1(13 \%)$ & 1,000 & $4(11 \%)$ & $2(18 \%)$ & 0,614 \\
\hline Desconocido & $4(10 \%)$ & $3(38 \%)$ & 0,084 & $3(8 \%)$ & $4(36 \%)$ & 0,042 \\
\hline
\end{tabular}


idarrubicina y citarabina y $6 \%(\mathrm{n}=3)$ azacitidina (todos pacientes mayores de 70 años). Las dosis promedio de las drogas utilizadas fueron: citarabina $127 \mathrm{mg} / \mathrm{m}^{2}$ (rango: 56-200) y daunorrubicina $74 \mathrm{mg} / \mathrm{m}^{2}$ (rango: $30-100$ ). El 25\% restante de los pacientes $(n=16)$ se manejó paliativamente. En la Tabla 1 se resumen las características clínicas, laboratorio y citogenéticas según si recibieron o no quimioterapia.

La RC a la inducción (RC1) se logró en 70,2\% de los pacientes $(\mathrm{n}=33)$ siendo $65 \%$ en el grupo que recibió daunorrubicina $<90 \mathrm{mg} / \mathrm{m}^{2}(\mathrm{n}=11)$ y de $81 \%(n=17)$ en aquellos con dosis de $90 \mathrm{mg} /$ $\mathrm{m}^{2}$ de daunorrubicina $(\mathrm{p}=0,293)$.

En la Tabla 3 se exponen el porcentaje de pacientes que logró remisión completa según el esquema de inducción usado. Del punto de vista estadístico podemos señalar lo siguiente: Idarrubicina vs daunorrubicina $<90 \mathrm{mg} / \mathrm{m}^{2} ; \mathrm{p}=0,555$; Idarrubicina vs daunorrubicina $>90 \mathrm{mg} / \mathrm{m}^{2}$ : $\mathrm{p}=0,580$; idarrubicina vs azacitidina: $=0,107$.

La mortalidad en inducción fue 4,2\% $(\mathrm{n}=2)$ por causas infecciosas.

Tabla 3. Remisión completa lograda por los pacientes según el esquema de inducción usado

\begin{tabular}{|lcc|}
\hline $\begin{array}{l}\text { Esquema } \\
\text { inducción }\end{array}$ & $\begin{array}{c}\text { Total } \\
\text { pacientes }\end{array}$ & $\begin{array}{c}\text { Remisión } \\
\text { completa } \mathbf{n ~ ( \% ) ~}\end{array}$ \\
\hline $7+3$ (dauno $<90)$ & 17 & $11(65)$ \\
\hline $7+3$ (dauno $>90)$ & 21 & $17(81)$ \\
\hline $7+3$ (ida) & 5 & $5(100)$ \\
\hline Azacitidina & 3 & $1(33)$ \\
\hline
\end{tabular}

\section{Quimioterapia de postremisión o consolidación}

En 75\% $(\mathrm{n}=35)$ de los pacientes que recibieron QT de inducción, se administró QT de consolidación con los siguientes esquemas: 19 pacientes con HiDAC (dosis promedio: $2,2 \mathrm{~g} / \mathrm{m}^{2}$ por 2,5 ciclos), 2 pacientes con azacitidina y 14 pacientes con esquema HAM (citarabina dosis promedio: $1,8 \mathrm{~g} / \mathrm{m}^{2}$ por 2,5 ciclos más mitoxantrona $10 \mathrm{mg} / \mathrm{m}^{2}$ ).

En la Tabla 4 se resumen las complicaciones más frecuentes, SLR y SG de cada esquema usado.

El 23,5\% $(n=11)$ restante del grupo no recibió QT de consolidación, todos pacientes mayores de 66 años que no lograron RC.

Del total de pacientes tratados, $15 \%(n=7)$ procedieron a trasplante de células hematopoyéticas $(\mathrm{TCH}): 4$ por riesgo citogenético desfavorable; 1 por persistencia de inversión del cromosoma 16 post 3 ciclos de consolidación y 2 por recaída post QT.

Los pacientes con riesgo citogenético desfavorable al diagnóstico procedieron a trasplante en primera remisión completa.

\section{Complicaciones de la quimioterapia}

Del total de pacientes tratados, $87 \%(\mathrm{n}=41)$ presentó neutropenia febril en la quimioterapia de inducción de focos pulmonar e intestinal principalmente. Destaca dentro de la etiología de los gérmenes aislados la presencia de hongos en $17 \%$ ( $\mathrm{n}=7$; Aspergillus sp, Mucor sp, Candida sp).

\section{Sobrevida y mortalidad en el seguimiento}

La SLR fue de $25 \%$ y la SG de $31 \%$ en los pacientes que recibieron QT (Figura 1a). No se evidenció una diferencia estadísticamente signi-

\section{Tabla 4. Sobrevida global (SG) media, a 3 años, recaída y causas de muerte según esquema de quimioterapia de consolidación usado}

\begin{tabular}{|lccccc|}
\hline Esquema & $\begin{array}{c}\text { Total pacientes } \\
\text { (n) }\end{array}$ & $\begin{array}{c}\text { Recaída } \\
\text { (\%) }\end{array}$ & SG media & $\begin{array}{c}\text { SG a 3 años } \\
\text { (\%) }\end{array}$ & $\begin{array}{c}\text { Causas de muerte } \\
\text { (\%) }\end{array}$ \\
\hline HiDAC & 19 & 21 & 24 meses & 68,4 & $\begin{array}{l}50=1 \text { leucemia } \\
50=\text { sepsis }\end{array}$ \\
HAM & 14 & 71 & 15 meses & 35,8 & $\begin{array}{l}80=1 \text { leucemia } \\
20=\text { sepsis }\end{array}$ \\
Azacitidina & 2 & & & 0 & $\begin{array}{l}50=\text { leucemia } \\
50=\text { sepsis }\end{array}$ \\
\hline
\end{tabular}

HiDAC: dosis altas de citrarabina. HAM: dosis altas de citarabina con mitoxantrona. 
ficativa en la SG de los pacientes tratados con QT mayores y menores de 60 años (Figura 1b).

Al comparar la mediana de SG del grupo tratado con QT con el grupo que no recibió QT, ésta fue de 31 meses (rango: 0-53 meses) y de 1 mes (rango: 0-6 meses), respectivamente $(\mathrm{p}<0,01)$

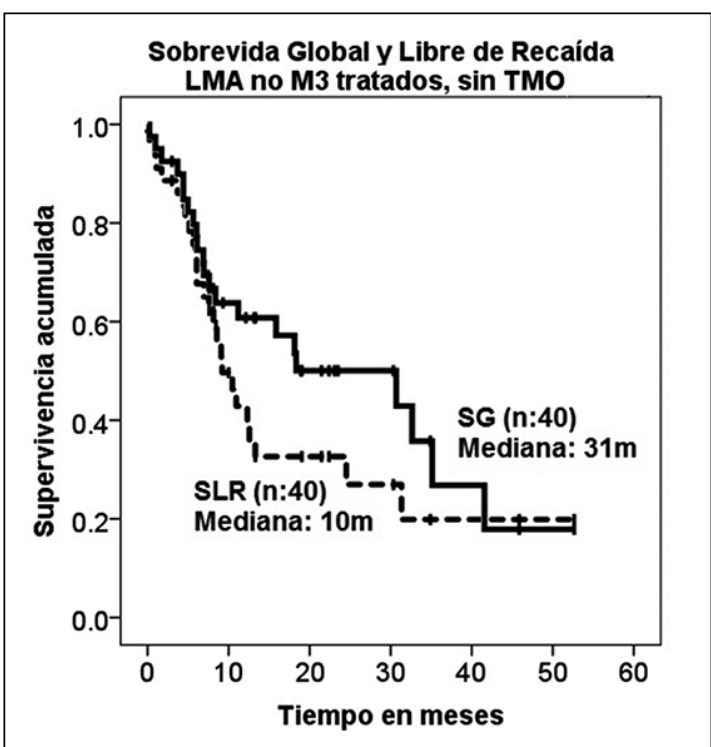

Figura 1a. SG y sobrevida libre de recurrencia (SLR) en pacientes tratados con quimioterapia por LMA.

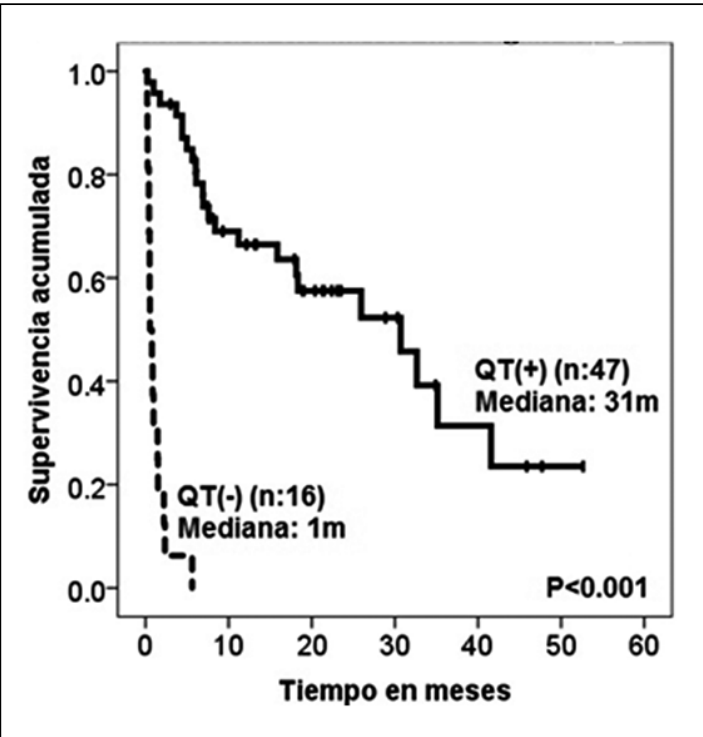

Figura 2a. SG según uso no de quimioterapia en LMA.
(Figura 2a). De la misma manera, los pacientes que solamente recibieron QT inducción (y no consolidación) presentaron una sobrevida significativamente mayor comparados con aquellos que no recibieron inducción (6 meses vs 1 mes, respectivamente, $\mathrm{p}<0,001$ ) (Figura $2 \mathrm{~b}$ ).

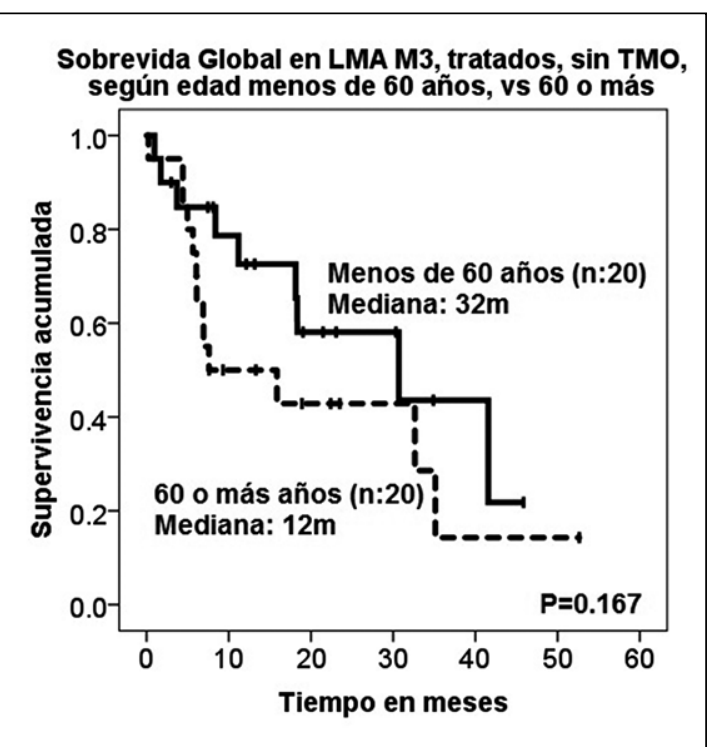

Figura 1b. SG según edad al diagnóstico de LMA.

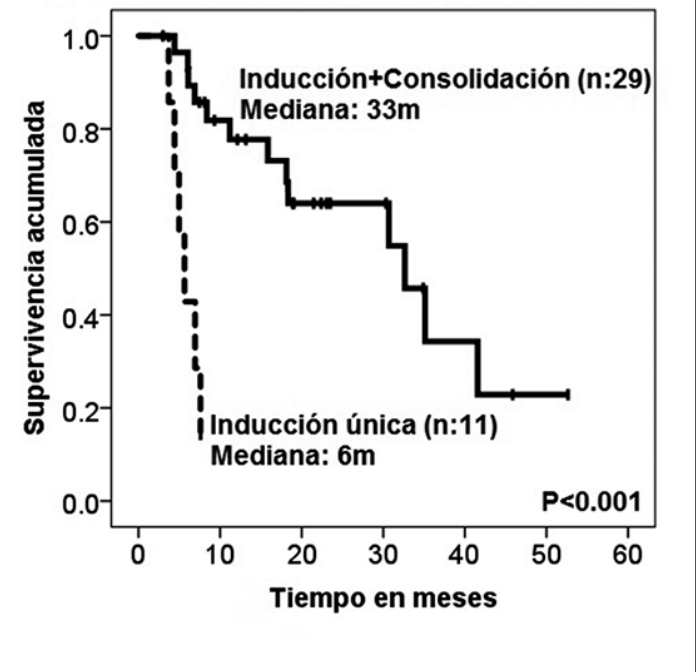

Figura 2b. SG según uso de quimioterapia de inducción vs inducción + consolidación. 


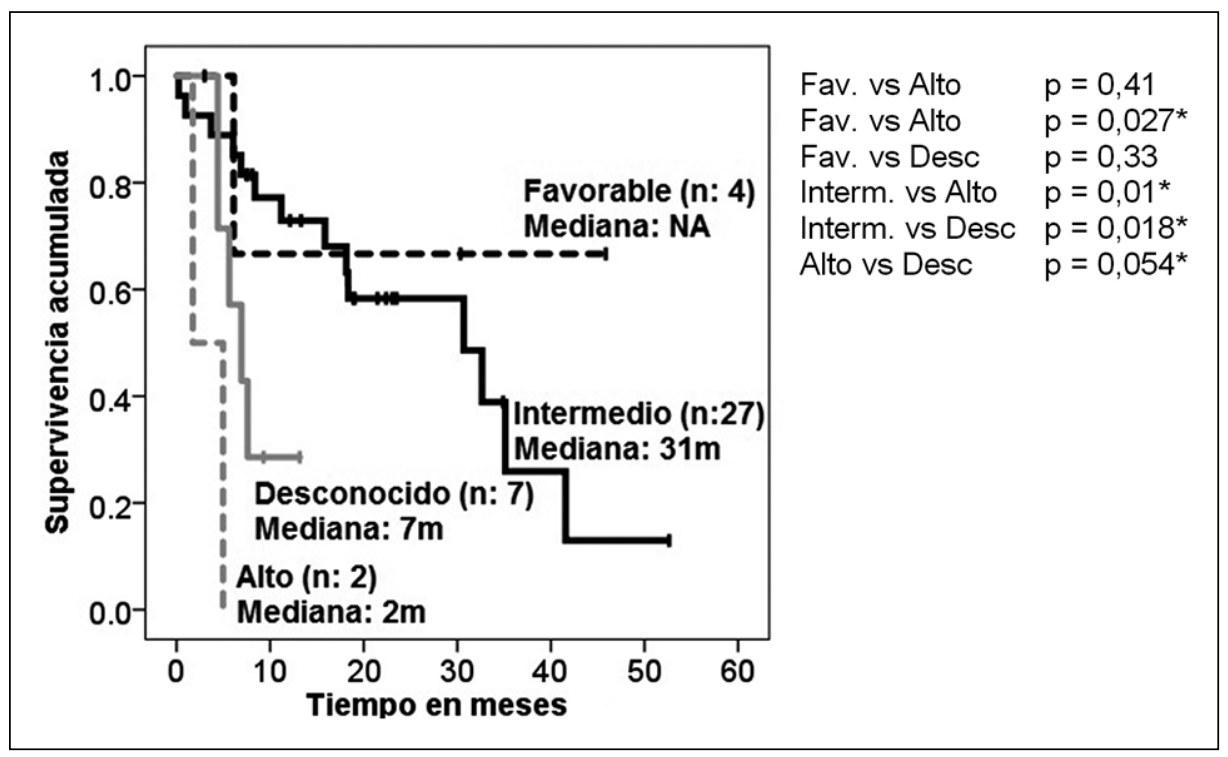

Figura 3. SG según riesgo citogenético.

El riesgo citogenético también tuvo una asociación significativa con sobrevida al comparar los 4 grupos identificados, con mejor sobrevida en el grupo favorable comparado con los otros grupos de riesgo (Figura 3 ).

La mortalidad durante el seguimiento en el grupo de pacientes que sobrevivió a la QT de inducción, fue $47 \%(\mathrm{n}=22), 2 / 3$ falleciendo por leucemia y $1 / 3$ por causas infecciosas.

\section{Análisis multivariado}

El análisis multivariado demostró que las variables que influyeron en forma significativa en la SLR y SG de los pacientes tratados fueron: obtención de remisión completa, utilización de QT de consolidación (HiDAC vs otras) y riesgo citogenético $(\mathrm{p}<0,05)($ Tabla 5$)$.

\section{Discusión}

La LMA es la leucemia más frecuente en adultos con una incidencia en aumento proporcional al envejecimiento de la población. Nuestro análisis mostró que dentro de las características clínicas y de laboratorio destacaban la edad media de 55,4 años, siendo menor a la descrito internacionalmente $^{8}$ (67 años) $)^{9}$ y mayor a los datos nacionales publicados $^{3}$ (48 años) ${ }^{4}$. La mayoría de los pacientes
Tabla 5. Análisis multivariado por método de regresión de Cox. Variables que influyeron o no en forma significativa en la SG

\begin{tabular}{|lc|}
\hline Variable & Valor $\mathbf{p}$ \\
\hline Edad al diagnóstico & 0,106 \\
\hline Recuento de leucocitos & 0,775 \\
\hline Porcentaje blastos S.P & 0,579 \\
\hline Nivel de hemoglobina & 0,453 \\
\hline Recuento de plaquetas & 0,446 \\
\hline Porcentaje blastos M.O & 0,156 \\
\hline Riesgo citogénetico & $\mathbf{0 , 0 0 9}$ \\
\hline Lograr Remisión Completa & $\mathbf{0 , 0 3 6}$ \\
\hline Consolidación (sí vs no) & $\mathbf{0 , 0 0 1}$ \\
\hline
\end{tabular}

se presentaron con bicitopenia y blastos en sangre periférica, salvo 9,5\% en los pacientes en que no se observaron células inmaduras en el hemograma. El 51\% de los pacientes fueron catalogados como riesgo intermedio citogenético lo cual es concordante con lo publicado en la literatura ${ }^{9,10 .}$

En relación al tratamiento, la quimioterapia combinada es la modalidad actual de tratamiento usado en pacientes con LMA con regímenes de inducción tipo " $7+3$ " con el cual se lograrían tasas 
de RC entre 70-80\% de los casos con un solo curso de $\mathrm{QT}^{3}$. En los pacientes tratados en nuestro centro se logró la RC en $70 \%$, destacando que en aquellos que se usó dosis altas de daunorrubicina (90 $\left.\mathrm{mg} / \mathrm{m}^{2}\right)$, la tasa de RC fue más alta $(81 \%)$ lo cual coincide con lo publicado previamente, en cuanto a la eficacia de las dosis altas de daunorrubicina ${ }^{11}$ Si bien encontramos que $100 \%$ de los pacientes que usaron idarrubicina logran la remisión completa esto no es significativo dado el bajo número de pacientes de este grupo. Además encontramos que el uso de azacitina en inducción no logró respuestas comparables con la quimioterapia estándar pero fue una opción de tratamiento en pacientes con comorbilidades y contraindicaciones para un tratamiento agresivo.

Si bien la QT de inducción es altamente efectiva, presenta toxicidades importantes a considerar que pueden influir en la morbimortalidad de nuestros pacientes tales como citopenias, infecciones, hemorragias, síndrome de lisis tumoral, alteraciones hidroelectrolíticas y otras complicaciones $^{10-13}$. En nuestra serie encontramos una alta incidencia de infecciones fúngicas como causal de la neutropenia febril post quimioterapia de inducción, lo cual fue superior a lo reportado ${ }^{14}$. Como posibles explicaciones a este fenómeno se podría asociar a una selección positiva por uso de profilaxis antimicrobiana y a que la profilaxis antifúngica empleada actualmente no es la adecuada.

Encontramos una baja tasa de mortalidad en inducción de 4,2\% lo cual puede estar reflejando un adecuado tratamiento interdisciplinario de los pacientes, acceso a mejores antibióticos, y al traslado precoz de estos pacientes a la unidad de cuidados intensivos.

Una vez lograda la RC, sin terapia citotóxica posterior, virtualmente todos los pacientes recaerán dentro de una media de 4 meses ${ }^{15}$. Por el contrario, los pacientes jóvenes o adultos jóvenes que reciben QT de consolidación, logran tasas de SG mayores o igual a $44 \%$ a 4 años $^{16}$. Dentro de las opciones de terapia de post remisión en nuestros pacientes se usaron quimioterapia de consolidación $(75 \% \mathrm{n}=35)$ y TCH (no reportado en esta serie). El tratamiento quimioterápico más usado de consolidación fue HiDAC.

En el análisis multivariado por método de regresión de Cox se demostró que las variables que influyeron en forma significativa en la SLR y SG de los pacientes tratados fueron: obtención de remisión, utilización de QT de consolidación (citarabina en dosis altas vs otras) y el riesgo citogenético.

En el seguimiento destacan la SLR 25\% y SG de $31 \%$ en los pacientes que recibieron QT. Lo anterior señala que se necesitan mejores tratamientos de consolidación para pacientes con LMA, más allá de la quimioterapia tradicional actualmente en uso.

No encontramos diferencias estadísticamente significativas en la SG de los pacientes tratados con QT al comparar mayores y menores de 60 años. Ésto podría deberse al número bajo de pacientes sobre ese rango de edad o bien al buen estado funcional de los pacientes que consideramos candidatos para recibir la QT.

Otro punto importante de destacar en el grupo de pacientes mayores de 60 años, es el beneficio de recibir sólo inducción (y no consolidación) versus manejo paliativo, pues encontramos una diferencia estadísticamente significativa de SG de 6 meses versus 1 mes lo que sugiere que las decisiones de tratamiento deben ser individualizadas, tomando en cuenta la edad fisiológica más que cronológica basándose en el estado funcional y la presencia de factores conocidos de riesgo adverso como los citogenéticos. Al igual que otros estudios en la literatura internacional, el riesgo citogenético tuvo una asociación significativa con sobrevida al comparar los 4 grupos identificados ${ }^{13}$.

En conclusión, del presente estudio podemos señalar que nuestros resultados son similares a lo reportado en análisis internacionales en cuanto a mortalidad por quimioterapia, SLR y SG. Sin embargo, los resultados globales aún son pobres, lo que hace necesario plantear la necesidad de mejores acceso terapias de consolidación como el TCH.

\section{Referencias}

1. Smith A, Howell D, Patmore R, Jack A, Roman E. Incidence of haematological malignancy by sub-type: a report from the Haematological Malignancy Research Network. Br J Cancer 2011; 105: 1684-92.

2. Siegel R, Ma J, Zou Z, Jemal A. Cancer statistics, 2014. CA Cancer J Clin 2014; 64: 9-29.

3. Puga BL, Cabrera ME, Undurraga MS, Etcheverry R, Vacarezza R, Ducach G, et al. Acute myeloid leukemia in the adult. Results of the National Antineoplastic Drug 
Protocol at the Hospital del Salvador, 1990-1998]. Rev Med Chile 2000; 128: 1191-8.

4. Hou HA, Lin CC, Chou WC, Liu CY, Chen CY, Tang $\mathrm{JL}$, et al. Integration of cytogenetic and molecular alterations in risk stratification of 318 patients with de novo non-M3 acute myeloid leukemia. Leukemia 2014; 28 : 50-8.

5. Cheson BD, Bennett JM, Kopecky KJ, Büchner T, Willman CL, Estey EH, et al. Revised recommendations of the International Working Group for diagnosis, standardization of response criteria, treatment outcomes, and reporting standards for therapeutic trials in acute myeloid leukemia. J Clin Oncol 2003; 21 (24): 4642-9.

6. Freifeld AG, Bow EJ, Sepkowitz KA, Boeckh MJ, Ito JI, Mullen CA, et al. Clinical practice guideline for the use of antimicrobial agents in neutropenic patients with cancer: 2010 update by the infectious diseases society of america. Clin Infect Dis 2011; 52: e56-93.

7. Floud S. Continuing the pursuit of a common language-the sixth edition of A Dictionary of Epidemiology. Eur J Clin Invest 2014; 44: 1121.

8. http://ash-sap.hematologylibrary.org/content/2013/481.

9. Byrd JC, Mrózek K, Dodge RK, Carroll AJ, Edwards CG, Arthur DC, et al. Pretreatment cytogenetic abnormalities are predictive of induction success, cumulative incidence of relapse, and overall survival in adult patients with de novo acute myeloid leukemia: results from Cancer and Leukemia Group B (CALGB 8461). Blood 2002; 100: 4325-36.
10. Döhner H, Estey EH, Amadori S, Appelbaum FR, Büchner T, Burnett AK, et al. Diagnosis and management of acute myeloid leukemia in adults: recommendations from an international expert panel, on behalf of the European Leukemia Net Blood 2010; 115: 453-74.

11. Fernández HF, Sun Z, Yao X, Litzow MR, Luger SM, Paietta EM, et al. Anthracycline dose intensificacion in acute myeloid leukemia. New England Journal of Medicine 2009; 361: 1249-59.

12. Slichter SJ, Kaufman RM, Assmann SF, McCullough J, Triulzi DJ, Strauss RG, et al. Dose of prophylactic platelet transfusions and prevention of hemorrhage. $\mathrm{N}$ Engl J Med 2010; 362: 600-13.

13. Zuckerman T, Ganzel C, Tallman MS, Rowe JM. How I treat hematologic emergencies in adults with acute leukemia. Blood 2012; 120: 1993-2002.

14. Nucci M, Anaissie E. How we treat invasive fungal diseases in patients with acute leukemia: the importance of an individualized approach. Blood, 18 December 2014. Volume 124, number 26.

15. Cassileth PA, Harrington DP, Hines JD, Oken MM, Mazza JJ, McGlave P, et al. Maintenance chemotherapy prolongs remission duration in adult acute nonlymphocytic leukemia. J Clin Oncol 1988; 6: 583-7.

16. Mayer RJ, Davis RB, Schiffer CA, Berg DT, Powell BL, Schulman $\mathrm{P}$, et al. Intensive postremission chemotherapy in adults with acute myeloid leukemia. N Engl J Med 1994; 361: 896-903. 